\title{
Photon propagation in nontrivial backgrounds
}

\author{
Palash B. Pal \\ Physics Department, University of Calcutta, 92 APC Road, Calcutta 700009, India
}

(Received 30 May 2020; accepted 22 July 2020; published 6 August 2020)

\begin{abstract}
Propagation of photons (or of any spin-1 boson) is of interest in different kinds of nontrivial background, including a thermal bath, or a background magnetic field, or both. We give a unified treatment of all such cases, casting the problem as a matrix eigenvalue problem. The matrix in question is not a normal matrix, and therefore care should be given to distinguish the right eigenvectors from the left eigenvectors. The polarization vectors are shown to be right eigenvectors of this matrix, and the polarization sum formula is seen as the completeness relation of the eigenvectors. We show how this method is successfully applied to different nontrivial backgrounds.
\end{abstract}

DOI: 10.1103/PhysRevD.102.036004

\section{INTRODUCTION}

Propagation of electromagnetic waves through any background is a subject of huge interest. In the language of quantum theory, we rename this subject as the question of photon propagation. Using the methods of quantum field theory in thermal background, the question of photon propagation was analyzed in a thermal background $[1,2]$, and well-known attributes of material medium like the dielectric constant and the magnetic permeability were identified in the framework of the quantum field theoretical treatment. It was also shown how to extend this analysis to chiral media [3] and describe natural optical activity in quantum field theoretic terms. The propagation of photons in pure magnetic fields was discussed later [4-6], and an expression for the Faraday effect was derived in terms of form factors that appear in the quantum theoretical framework. The more general case of a magnetic field in a medium, combining the two kinds of backgrounds mentioned earlier, has also been a subject of great interest $[4,7]$. In many cases the discussion does not explicitly mention photons, but rather gluons [8-10] or even $\rho$-mesons [11], but that makes no difference in the general structure of the problem. Basically, it is the problem of the propagation of a spin-1 boson in a nontrivial background.

For each kind of background, one introduces appropriate parameters and notations, and obtains the photon propagator and dispersion relations from there. What we propose to do in this paper is to develop a unified approach that works for all kinds of background. This will give us

Published by the American Physical Society under the terms of the Creative Commons Attribution 4.0 International license. Further distribution of this work must maintain attribution to the author(s) and the published article's title, journal citation, and DOI. Funded by SCOAP. important insight into the question of photon propagation, which we will then apply to specific backgrounds, rediscovering some of the old formulas with the new insight, and finding expressions for the polarization vectors which are sometimes difficult to find from the usual approach.

\section{THE SELF-ENERGY FUNCTION}

The momentum-space Lagrangian of a system is related to the action by the relation

$$
\mathcal{A}=\int \frac{d^{4} k}{(2 \pi)^{4}} \mathcal{L}(k)
$$

For the photon field in the vacuum, the momentum-space Lagrangian is given by

$$
\mathcal{L}_{0}(k)=-\frac{1}{2} k^{2} \tilde{\eta}_{\mu \nu} A^{\mu}(-k) A^{\nu}(k)-A^{\mu}(-k) j_{\mu}(k),
$$

where

$$
\tilde{\eta}_{\mu \nu}=\eta_{\mu \nu}-\frac{k_{\mu} k_{\nu}}{k^{2}}
$$

Quantum corrections in the vacuum give an extra contribution which has the same generic form, and therefore can be absorbed in the definition of the photon field. In a nontrivial background, however, the photon Lagrangian will have nontrivial contributions, and will therefore be modified to

$$
\mathcal{L}=\mathcal{L}_{0}+\mathcal{L}^{\prime},
$$

where the quadratic part of $\mathcal{L}^{\prime}$ can be written as

$$
\mathcal{L}^{\prime}(k)=\frac{1}{2} \Pi_{\mu \nu}(k) A^{\mu}(-k) A^{\nu}(k)
$$


Here, $\Pi_{\mu \nu}(k)$ represents the self-energy function. In addition to the momentum $k$, it can depend on other parameters that characterize the background. Those parameters depend on the nature of the background, and are not shown here in order to maintain generality.

In order to proceed, we need to know some properties of the self-energy function. We itemize them now.

Gauge invariance: Gauge invariance says that adding a term proportional to $k^{\mu}$ to the photon field should not have any physical implication. This property gives the relations

$$
\begin{aligned}
& k^{\mu} \Pi_{\mu \nu}(k)=0, \\
& k^{\nu} \Pi_{\mu \nu}(k)=0 .
\end{aligned}
$$

Bose symmetry: Bose symmetry is the statement that the Lagrangian should be invariant under the interchange of the two photon field factors. Interchange of the two photon fields means the changes

$$
k \rightarrow-k, \quad \mu \leftrightarrow \nu .
$$

Clearly Eq. (2) satisfies this interchange trivially. For the extra contribution of Eq. (5), however, it implies that the self-energy function must satisfy the relation

$$
\Pi_{\mu \nu}(k)=\Pi_{\nu \mu}(-k) .
$$

So, $\Pi_{\mu \nu}$ need not be symmetric in its indices, as is often claimed. Equation (8) only says that in $\Pi_{\mu \nu}$, the terms symmetric in the indices should be even in $k$, whereas the antisymmetric terms should be odd [3].

Hermiticity: There is a constraint from the Hermiticity of the Lagrangian. Since the coordinate space version of $\mathcal{L}$ must be Hermitian, we must have

$$
[\mathcal{L}(k)]^{\dagger}=\mathcal{L}(-k) .
$$

The $\mathcal{L}_{0}$ part automatically satisfies this condition. To see what it implies for $\mathcal{L}^{\prime}$, let us first note that in the co-ordinate space, the field $A^{\mu}(x)$ is a Hermitian field, so that its Fourier transform satisfies the relation

$$
\left(A^{\mu}(-k)\right)^{\dagger}=A^{\mu}\left(k^{*}\right) .
$$

In this paper, we discuss only the dispersive part of the self-energy for which $k$ is real. Then, taking the Hermitian conjugate of Eq. (5), we obtain

$$
\begin{aligned}
{\left[\mathcal{L}^{\prime}(k)\right]^{\dagger} } & =\frac{1}{2}\left(\Pi_{\mu \nu}(k)\right)^{*}\left(A^{\mu}(-k)\right)^{\dagger}\left(A^{\nu}(k)\right)^{\dagger} \\
& =\frac{1}{2}\left(\Pi_{\mu \nu}(k)\right)^{*} A^{\mu}(k) A^{\nu}(-k) .
\end{aligned}
$$
get

Imposing the condition of Eq. (9) on the $\mathcal{L}^{\prime}$ part, we

$$
\left(\Pi_{\mu \nu}(k)\right)^{*}=\Pi_{\mu \nu}(-k),
$$

comparing Eq. (5) with Eq. (11).

We can summarize the results of Eqs. (8) and (12) by writing

$$
\Pi_{\mu \nu}(-k)=\left(\Pi_{\mu \nu}(k)\right)^{*}=\Pi_{\nu \mu}(k) .
$$

Any tensor can be written as a sum of a symmetric and an antisymmetric tensor. Equation (13) says that, for the dispersive part,

(1) The symmetric part of $\Pi_{\mu \nu}$ would be real and an even function of $k$.

(2) The antisymmetric part of $\Pi_{\mu \nu}$ would be purely imaginary, and odd in $k$.

In order to explore properties of the self-energy function, it will be convenient to define a matrix $\Pi(k)$ whose element in the $\mu$ th row and $\nu$ th column is $\Pi^{\mu}{ }_{\nu}(k)$. Note that these are the objects with one up and one down index, not the objects that appear in Eq. (13). The object $\Pi_{\mu \nu}$ is the element in the $\mu$ th row and $\nu$ th column of the matrix $\boldsymbol{\eta} \Pi$, where $\boldsymbol{\eta}$ is a matrix whose element in the $\mu$ th row and $\nu$ th column is $\eta_{\mu \nu}$. In the matrix notation, Eq. (13) becomes

$$
[\boldsymbol{\eta} \boldsymbol{\Pi}(-k)]^{\top}=[\boldsymbol{\eta} \boldsymbol{\Pi}(k)]^{\dagger}=\boldsymbol{\eta} \boldsymbol{\Pi}(k),
$$

It will be easier to understand these equations if we write them in terms of the matrix elements. For that, we only need to raise the index $\mu$ in Eq. (13). Since

$$
\Pi_{\nu}{ }^{\mu}=\eta_{\nu \alpha} \Pi_{\beta}^{\alpha} \eta^{\beta \mu},
$$

we obtain

$$
\Pi_{\nu}^{\mu}(-k)=\left(\Pi_{\nu}^{\mu}(k)\right)^{*}=\eta_{\nu \alpha} \Pi_{\beta}^{\alpha}(k) \eta^{\beta \mu},
$$

or, more explicitly,

$$
\begin{gathered}
\Pi_{0}^{0}(-k)=\left[\Pi_{0}^{0}(k)\right]^{*}=\Pi_{0}^{0}(k), \\
\Pi_{j}^{0}(-k)=\left[\Pi_{j}^{0}(k)\right]^{*}=-\Pi_{0}^{j}(k), \\
\Pi_{j}^{i}(-k)=\left[\Pi_{j}^{i}(k)\right]^{*}=\Pi_{i}^{j}(k) .
\end{gathered}
$$

These equations clearly tell us which elements of the matrix $\Pi$ are real and even functions of $k$, and which are imaginary and odd function of $k$. The implication of these conditions on various form factors appearing in the selfenergy will be indicated when we talk about specific backgrounds in Sec. V. 


\section{POLARIZATION VECTORS}

So far, we have not interpreted the gauge invariance condition in the matrix language. We now notice that Eq. (6b) can be written as

$$
\Pi^{\mu}{ }_{\nu} k^{\nu}=0 .
$$

In the matrix notation, this equation becomes

$$
\Pi k=0,
$$

where $\boldsymbol{k}$ is the column matrix whose elements are $k^{\mu}$. This is an eigenvalue equation, implying that $\boldsymbol{k}$ is an eigenvector of the matrix $\boldsymbol{\Pi}$, and the corresponding eigenvalue is zero.

This fact prompts us to look at the eigensystem of $\Pi$. We can define eigenvectors of this matrix through the relation

$$
\Pi \epsilon_{A}=\Lambda_{A} \epsilon_{A}
$$

where the $\Lambda_{A}$ 's are eigenvalues. Note that, although we have used the convention of implied summation on Lorentz indices earlier, there is no implied sum on the index that labels the eigenvectors, here or elsewhere in this article. Also, it does not matter whether this index appears as subscript or superscript: they mean the same thing. We just put the index wherever it is convenient in any formula.

To be precise, Eq. (20) tells us that the $\boldsymbol{\epsilon}_{A}$ 's are the right eigenvectors of the matrix П. To check what the left eigenvectors are, we multiply Eq. (20) from the left by the matrix $\boldsymbol{\eta}$ and take the Hermitian conjugate of the resulting equation to obtain

$$
\boldsymbol{\epsilon}_{A}^{\dagger}(\boldsymbol{\eta} \boldsymbol{\Pi})^{\dagger}=\Lambda_{A}^{*}\left(\boldsymbol{\eta} \boldsymbol{\epsilon}_{A}\right)^{\dagger} .
$$

We can now use Eq. (14), and write this equation in the form

$$
\left(\boldsymbol{\eta} \boldsymbol{\epsilon}_{A}\right)^{\dagger} \boldsymbol{\Pi}=\Lambda_{A}^{*}\left(\boldsymbol{\eta} \boldsymbol{\epsilon}_{A}\right)^{\dagger},
$$

using $\boldsymbol{\eta}=\boldsymbol{\eta}^{\dagger}$.

Equation (22) is also an eigenvalue equation, except that here the eigenvectors multiply the matrix from the left. We see two things from this equation. First, we see that the eigenvalues $\Lambda_{A}$ must be real, since they are defined as the solution of the equation

$$
\operatorname{det}(\boldsymbol{\Pi}-\Lambda \mathbf{1})=0,
$$

an equation which makes no reference to the right or left eigenvectors. Second, we see that, corresponding to a particular eigenvalue $\Lambda_{A}$, the right eigenvector $\boldsymbol{R}_{A}$ and the left eigenvector $\boldsymbol{L}_{A}$ are related by the matrix $\boldsymbol{\eta}$ :

$$
\boldsymbol{R}_{A}=\boldsymbol{\epsilon}_{A} \quad \Rightarrow \quad \boldsymbol{L}_{A}=\boldsymbol{\eta} \boldsymbol{\epsilon}_{A} .
$$

It is not surprising that the right and left eigenvectors are not the same. In fact, this is expected for any matrix which is not normal, i.e., which does not commute with its Hermitian conjugate. Since $\boldsymbol{\Pi}$ is not a normal matrix, some properties of the left and right eigenvectors of such matrices are worth summarizing here [12].

Let us adopt a more general notation, and write the right and left eigenvector equations of a matrix $\boldsymbol{M}$ as

$$
\begin{gathered}
\boldsymbol{M R}_{A}=\mu_{A} \boldsymbol{R}_{A}, \\
\boldsymbol{L}_{B}^{\dagger} \boldsymbol{M}=\mu_{B} \boldsymbol{L}_{B}^{\dagger} .
\end{gathered}
$$

If we multiply the first equation by $\boldsymbol{L}_{B}^{\dagger}$ from the left and the second equation by $\boldsymbol{R}_{A}$ from the right, the left sides of the two resulting equations will be the same, implying the following relation involving the right sides:

$$
\left(\mu_{A}-\mu_{B}\right) \boldsymbol{L}_{B}^{\dagger} \boldsymbol{R}_{A}=0 .
$$

Thus, $\boldsymbol{L}_{B}^{\dagger} \boldsymbol{R}_{A}=0$ if $\mu_{A} \neq \mu_{B}$, and we can choose the right and the left eigenvectors in a way that the equation

$$
\boldsymbol{L}_{B}^{\dagger} \boldsymbol{R}_{A}=\zeta_{A} \delta_{A B}
$$

holds, where the $\zeta_{A}$ 's are normalization constants.

The second important property concerns the matrix defined as

$$
\boldsymbol{X}=\sum_{A} \frac{1}{\zeta_{A}} \boldsymbol{R}_{A} \boldsymbol{L}_{A}^{\dagger}
$$

Clearly, using Eq. (27), we see that

$$
\begin{gathered}
\boldsymbol{X} \boldsymbol{R}_{B}=\boldsymbol{R}_{B}, \\
\boldsymbol{L}_{B}^{\dagger} \boldsymbol{X}=\boldsymbol{L}_{B}^{\dagger} .
\end{gathered}
$$

This means that all eigenvalues of $\boldsymbol{X}$ are equal to 1 , implying that $\boldsymbol{X}$ is the identity matrix:

$$
\sum_{A} \frac{1}{\zeta_{A}} \boldsymbol{R}_{A} \boldsymbol{L}_{A}^{\dagger}=\mathbf{1}
$$

A particularly useful outcome of this relation can be seen by multiplying by $\boldsymbol{M}$ from the left side, and using Eq. (25) to obtain

$$
\boldsymbol{M}=\sum_{A} \frac{\mu_{A}}{\zeta_{A}} \boldsymbol{R}_{A} \boldsymbol{L}_{A}^{\dagger}
$$

It shows that a matrix can be written in terms of its eigenvectors and eigenvalues. 
Let us now leave the general discussion and return to the matrix $\Pi$ obtained from the self-energy. We have already noticed that $\boldsymbol{k}$ is one of its right eigenvectors. We denote this fact by writing

$$
\boldsymbol{\epsilon}_{(0)} \propto \boldsymbol{k},
$$

or, in component notation,

$$
\epsilon_{(0)}^{\mu} \propto k^{\mu}
$$

Leaving this one aside, there are three more eigenvectors. These are the polarization vectors, which we will denote by $\boldsymbol{\epsilon}_{a}$, with a lowercase roman index that runs from 1 to 3 . The corresponding left eigenvectors will be $\boldsymbol{\eta} \boldsymbol{\epsilon}_{a}$.

Equation (27) now implies the relation

$$
\boldsymbol{k}^{\dagger} \boldsymbol{\eta} \boldsymbol{\epsilon}_{a}=0 .
$$

In indexed notation, this reads

$$
k^{\mu} \epsilon_{\mu}^{a}=0 .
$$

There is also the relation of orthogonality of the polarization vectors. If the momentum vector $k^{\mu}$ is a timelike vector, the polarization vectors must be spacelike, and we can impose

$$
\boldsymbol{\epsilon}_{a}^{\dagger} \boldsymbol{\eta} \boldsymbol{\epsilon}_{b}=-\delta_{a b},
$$

which amounts to the choice

$$
\zeta_{a}=-1 \quad \text { for } a=1,2,3
$$

In indexed notation, Eq. (36) means

$$
\left(\epsilon_{a}^{\mu}\right)^{*} \epsilon_{\mu}^{b}=-\delta_{a b},
$$

and Eq. (31) gives

$$
\boldsymbol{\Pi}=-\sum_{a} \Lambda_{a} \boldsymbol{\epsilon}_{a}\left(\boldsymbol{\eta} \boldsymbol{\epsilon}_{a}\right)^{\dagger} .
$$

The $\Lambda_{a}$ 's are the eigenvalues of $\boldsymbol{\Pi}$, as defined earlier. Note that the sum is only on the three polarization vectors. The other eigenvector does not contribute since it is associated with a null eigenvalue. In the indexed notation, we can write the last equation as

$$
\Pi_{\nu}^{\mu}=-\sum_{a} \Lambda_{a} \epsilon_{a}^{\mu}\left(\epsilon_{\nu}^{a}\right)^{*} .
$$

This relation is easier to deal with using the matrix $\boldsymbol{\eta} \Pi$ which has both lower indices. We write

$$
\Pi_{\rho \nu}=\sum_{a} \Lambda_{a} P_{\rho \nu}^{a},
$$

where

$$
P_{\rho \nu}^{a}=-\epsilon_{\rho}^{a}\left(\epsilon_{\nu}^{a}\right)^{*} .
$$

Note that these objects satisfy the relation

$$
\eta^{\nu \lambda} P_{\mu \nu}^{a} P_{\lambda \rho}^{b}=\delta_{a b} P_{\mu \rho}^{a} .
$$

So the $P_{a}$ 's are, in some sense, projectors for the different polarization vectors. Because of Eq. (35), they satisfy the relations

$$
\begin{aligned}
& k^{\mu} P_{\mu \nu}^{a}=0, \\
& k^{\nu} P_{\mu \nu}^{a}=0 .
\end{aligned}
$$

Note that these equations are exactly similar to those in Eq. (6). This must be the case since $\Pi_{\mu \nu}$ is a linear superposition of these projection tensors, as shown in Eq. (41).

Equation (30) provides more information about the polarization vectors. Applying it on the matrix $\Pi$, we obtain the relation

$$
\frac{k^{\mu} k_{\nu}}{k^{2}}-\sum_{a} \epsilon_{a}^{\mu}\left(\epsilon_{\nu}^{a}\right)^{*}=\delta_{\nu}^{\mu},
$$

which can be rewritten as

$$
\sum_{a} P_{\mu \nu}^{a}=\tilde{\eta}_{\mu \nu}
$$

This is the polarization sum formula.

\section{PROPAGATOR AND DISPERSION RELATIONS}

The equation of motion of the photon field that follows from Eq. (4) is

$$
\left[-k^{2} \tilde{\eta}_{\mu \nu}+\Pi_{\mu \nu}(k)\right] A^{\nu}(k)=j_{\mu}(k),
$$

where $j^{\mu}$ is the current that the photon couples to. The equation for the propagator, after adding a gauge fixing term, is

$$
\left[-k^{2} \tilde{\eta}_{\mu \nu}+\Pi_{\mu \nu}(k)+\frac{1}{\xi} \frac{k_{\mu} k_{\nu}}{k^{2}}\right] D^{\nu \rho}=\delta_{\mu}^{\rho} .
$$

Substituting $\tilde{\eta}_{\mu \nu}$ from Eq. (46) and using the expression of Eq. (41) for $\Pi_{\mu \nu}$, we obtain the following equation that defines the propagator: 


$$
\left[\sum_{a}\left(-k^{2}+\Lambda_{a}\right) P_{\mu \nu}^{a}+\frac{1}{\xi} \frac{k_{\mu} k_{\nu}}{k^{2}}\right] D^{\nu \rho}=\delta_{\mu}^{\rho}
$$

It can now be easily seen that the propagator is given by

$$
\begin{aligned}
D^{\mu \nu}(k) & =-\sum_{a} \frac{P_{a}^{\mu \nu}}{k^{2}-\Lambda_{a}}+\frac{\xi}{k^{2}} \frac{k^{\mu} k^{\nu}}{k^{2}} \\
& =\sum_{a} \frac{\epsilon_{a}^{\mu} \epsilon_{a}^{\nu *}}{k^{2}-\Lambda_{a}}+\frac{\xi}{k^{2}} \frac{k^{\mu} k^{\nu}}{k^{2}}
\end{aligned}
$$

For the polarization $a$, the dispersion relation is the relation for which the propagator blows up, i.e.,

$$
k^{2}=\Lambda_{a} .
$$

It has to be remembered that $\Lambda_{a}$ is a function of the momentum. Thus, this is an implicit equation that has to be solved to obtain the dispersion relation. The polarization vector that satisfies this dispersion relation is $\epsilon_{a}^{\mu}$.

\section{EXAMPLES OF SPECIFIC BACKGROUNDS}

We will now illustrate how this formalism applies to various nontrivial backgrounds. We will always choose the photon momentum in the $x$-direction, i.e., in matrix form we will have

$$
\boldsymbol{k}=\left(\begin{array}{c}
\omega \\
K \\
0 \\
0
\end{array}\right)
$$

Then the components of the matrix $\tilde{\boldsymbol{\eta}}$ are given by

$$
\tilde{\boldsymbol{\eta}}=\frac{1}{k^{2}}\left(\begin{array}{cccc}
-K^{2} & \omega K & 0 & 0 \\
-\omega K & \omega^{2} & 0 & 0 \\
0 & 0 & k^{2} & 0 \\
0 & 0 & 0 & k^{2}
\end{array}\right)
$$

remembering that the matrix form corresponds to the components of the mixed tensor, whose first index is contravariant and the second index is covariant. Other tensors necessary for building up the self-energy tensor depend on the type of background, and will be defined as we go along.

\section{A. Thermal background}

A thermal background is characterized by a temperature and a chemical potential, both of which are scalars. There is one vector associated with a thermal medium, which is the center of mass velocity of the medium. Let us call it $u^{\mu}$. We can define

$$
\tilde{u}_{\mu}=\tilde{\eta}_{\mu \rho} u^{\rho}
$$

which satisfies the relation

$$
k^{\mu} \tilde{u}_{\mu}=0
$$

Using this, one can form the tensor

$$
L_{\mu \nu}=\frac{\tilde{u}_{\mu} \tilde{u}_{\nu}}{\tilde{u}^{2}}
$$

which vanishes when contracted with either $k^{\mu}$ or $k^{\nu}$. Therefore, it is a tensor that can be used for writing $\Pi_{\mu \nu}$. Of course $\tilde{\eta}_{\mu \nu}$ is another such tensor. So, we can write the self-energy as [1]

$$
\Pi_{\mu \nu}=a \tilde{\eta}_{\mu \nu}+b L_{\mu \nu},
$$

where $a$ and $b$ are Lorentz invariants.

The matrix $\Pi$ follows from Eq. (57) once we decide on the components of $u^{\mu}$. Let us work in a frame where the medium is at rest, so that the time component of $u^{\mu}$ is equal to 1 and all other components are zero. In this frame, we find

$$
\Pi=\frac{1}{k^{2}}\left(\begin{array}{cccc}
-(a+b) K^{2} & (a+b) \omega K & 0 & 0 \\
-(a+b) \omega K & (a+b) \omega^{2} & 0 & 0 \\
0 & 0 & a k^{2} & 0 \\
0 & 0 & 0 & a k^{2}
\end{array}\right) .
$$

Equation (17) implies that the form factors $a$ and $b$ are real, and are even functions of $k$.

It is easy to check that $\boldsymbol{k}$, defined in Eq. (52), is a right eigenvector and $\boldsymbol{\eta} \boldsymbol{k}$ is a left eigenvector of this matrix, with eigenvalue equal to zero. Among the others, there is a nondegenerate eigenvalue

$$
\Lambda_{(L)}=a+b,
$$

whose eigenvector is $\tilde{u}^{\mu}$. In matrix notation, we can write the eigenvector corresponding to this mode as

$$
\boldsymbol{\epsilon}_{(L)} \propto \tilde{\boldsymbol{u}},
$$

and we can define the projector corresponding to this mode as

$$
P_{\mu \nu}^{(L)}=L_{\mu \nu} .
$$

Since the spatial component of this polarization vector is along the direction of the photon momentum, this is called the longitudinal mode.

In compliance with Eq. (46), the sum of the projectors of the other two modes will be given by 


$$
P_{\mu \nu}^{(T)}=\tilde{\eta}_{\mu \nu}-P_{\mu \nu}^{(L)},
$$

and these two modes will be transverse and degenerate, with eigenvalues

$$
\Lambda_{(T)}=a .
$$

Following Eq. (50), we can now write down the photon propagator:

$$
D^{\mu \nu}(k)=-\frac{P_{(T)}^{\mu \nu}}{k^{2}-\Lambda_{(T)}}-\frac{P_{(L)}^{\mu \nu}}{k^{2}-\Lambda_{(L)}}+\frac{\xi}{k^{2}} \frac{k^{\mu} k^{\nu}}{k^{2}} .
$$

The dispersion relations of the different modes follow from Eq. (51).

\section{B. Chiral thermal medium}

It was pointed out $[3,13]$ that Eq. (57) is not the most general self-energy tensor that one can write using the two 4 -vectors $k^{\mu}$ and $u^{\mu}$. Rather, the general form would be

$$
\Pi_{\mu \nu}=a \tilde{\eta}_{\mu \nu}+b L_{\mu \nu}+i c C_{\mu \nu},
$$

where

$$
C_{\mu \nu}=\frac{1}{\kappa} \varepsilon_{\mu \nu \lambda \rho} k^{\lambda} u^{\rho} .
$$

where

$$
\kappa=\left|\sqrt{(k \cdot u)^{2}-k^{2}}\right|,
$$

a Lorentz invariant factor inserted to ensure that $C_{\mu \nu}$ is dimensionless, at par with the other tensors that appear in Eq. (65). With our choice of Eq. (52), $\kappa=|K|$.

Note that the extra term in the self-energy is not symmetric in the Lorentz indices. But this symmetry was never one of the requirements mentioned in Sec. II. The factor of $i$ accompanying this tensor in Eq. (65) ensures that the associated form factor $c$ is real, according to Eq. (12).

The matrix $\Pi$ can now be easily constructed, and it is

$\boldsymbol{\Pi}=\frac{1}{k^{2}}\left(\begin{array}{cccc}-(a+b) K^{2} & (a+b) \omega K & 0 & 0 \\ -(a+b) \omega K & (a+b) \omega^{2} & 0 & 0 \\ 0 & 0 & a k^{2} & i c k^{2} K / \kappa \\ 0 & 0 & -i c k^{2} K / \kappa & a k^{2}\end{array}\right)$.

Equation (17) shows that all three form factors, $a, b$, and $c$, are even functions of $k$, and all are real.

The longitudinal eigenvector and its eigenvalue are exactly the same as that found in the earlier case. But the transverse eigenvalues are no more degenerate. They, and their corresponding eigenvectors, are as follows:

$$
\Lambda_{( \pm)}=a \pm c K / \kappa, \quad \boldsymbol{\epsilon}_{( \pm)} \propto\left(\begin{array}{c}
0 \\
0 \\
1 \\
\mp i
\end{array}\right)
$$

Thus, the propagating modes are circularly polarized modes, and the left and right circular polarized waves have different dispersion relations $[3,13]$. The linearly polarized transverse waves are not eigenvectors of propagation. If one sends in a linearly polarized wave, its direction of polarization will rotate. This is the phenomenon of optical activity.

\section{Background magnetic field}

Optical activity can also be induced by a background magnetic field. This phenomenon is called the Faraday effect. In order to investigate it, we first need to find tensors built from the background field tensor $B_{\mu \nu}$ whose contraction vanishes with the photon momentum vector. Two such tensors were identified [4]:

$$
\begin{aligned}
& M_{\mu \nu}=\frac{1}{k^{2}} \varepsilon_{\mu \nu \sigma \tau} k^{\sigma} k_{\lambda} B^{\lambda \tau}, \\
& M_{\mu \nu}^{\prime}=B_{\mu \nu}-\frac{k_{\mu} k^{\lambda} B_{\lambda \nu}}{k^{2}}+\frac{k_{\nu} k^{\lambda} B_{\lambda \mu}}{k^{2}} .
\end{aligned}
$$

In this article, just to keep the formulas simple-looking, we will assume that the self-energy tensor does not have a term proportional to $M_{\mu \nu}^{\prime}$, i.e., we have

$$
\Pi_{\mu \nu}=a \tilde{\eta}_{\mu \nu}+i b M_{\mu \nu} .
$$

Note that we have put a factor of $i$ in the second term, in order to make sure that the form factor $b$ is real, as demanded from Eq. (13). It is also to be noted that the form factor $b$ has to be an odd function of $k$, which follows from Eq. (8).

In order to write the matrix $\boldsymbol{\Pi}$ explicitly, let us say that the background magnetic field is in the $x-y$ plane, making an angle $\beta$ with the $x$-axis. Then the only nonzero components of the background field tensor $B_{\mu \nu}$ are the following:

$$
\begin{aligned}
& B_{23}=-B_{32}=\cos \beta, \\
& B_{31}=-B_{13}=\sin \beta,
\end{aligned}
$$

where we have normalized the magnitude of the magnetic field to be unity. The matrix $\Pi$ that follows from Eq. (71) is then easily calculated: 


$$
\boldsymbol{\Pi}=\frac{1}{k^{2}}\left(\begin{array}{cccc}
-a K^{2} & a \omega K & -i b^{\prime} K^{2} & 0 \\
-a \omega K & a \omega^{2} & -i b^{\prime} \omega K & 0 \\
-i b^{\prime} K^{2} & i b^{\prime} \omega K & a k^{2} & 0 \\
0 & 0 & 0 & a k^{2}
\end{array}\right),
$$

where

$$
b^{\prime}=b \sin \beta \text {. }
$$

Note that if the magnetic field is parallel to the photon momentum, i.e., if $\sin \beta=0$, there is no effect. For what follows, we assume that $b^{\prime} \neq 0$, i.e., the magnetic field is not parallel to the photon momentum.

Apart from the trivial eigenvector which is the photon momentum, we can easily see that there is one eigenmode with

$$
\Lambda_{(3)}=a, \quad \boldsymbol{\epsilon}_{(3)}=\left(\begin{array}{l}
0 \\
0 \\
0 \\
1
\end{array}\right)
$$

This is the mode that is perpendicular to both the magnetic field and the photon 3-momentum. The other two eigenmodes can be easily solved, with the result:

$$
\Lambda_{( \pm)}=a \pm \frac{b^{\prime} K}{\sqrt{k^{2}}}, \quad \boldsymbol{\epsilon}_{( \pm)}=\left(\begin{array}{c}
K \\
\omega \\
\pm i \sqrt{k^{2}} \\
0
\end{array}\right) .
$$

These are in general elliptically polarized states. As in the case of chiral thermal medium, we see that the linearly polarized states are not eigenmodes of propagation. Thus, if a linearly polarized wave is sent through a magnetic field, its polarization vector will rotate as it moves. This is Faraday rotation [4].

\section{Magnetic field in a thermal medium}

We now go to the more complicated case where there is a thermal bath as well as a magnetic field. Because of the presence of the 4-vector $u^{\mu}$, we can define now [7] a 4 -vector for the magnetic field by the relation

$$
n^{\mu}=\frac{1}{2} \varepsilon^{\mu \nu \lambda \rho} u_{\nu} B_{\lambda \rho}
$$

It is easier to work with this vector rather than with the background field tensor.

We can define an associated vector

$$
\tilde{n}_{\mu}=\tilde{\eta}_{\mu \nu} n^{\nu}
$$

which satisfies the relation

$$
k^{\mu} \tilde{n}_{\mu}=0
$$

So, in $\Pi_{\mu \nu}$, we can have a term proportional to

$$
n_{\mu \nu}=\frac{\tilde{n}_{\mu} \tilde{n}_{\nu}}{\tilde{n}^{2}},
$$

because the said term will satisfy Eq. (6). There are other tensors which will satisfy Eq. (6). For example, one can consider

$$
S_{\mu \nu}=\frac{\tilde{u}_{\mu} \tilde{n}_{\nu}+\tilde{n}_{\mu} \tilde{u}_{\nu}}{\sqrt{\tilde{u}^{2} \tilde{n}^{2}}} .
$$

Detailed analysis of photon dispersion was made [7] with these four tensors. However, there can be many other tensors, including some which are antisymmetric in the indices. The list is quite long [4]. Here, we will take one of the antisymmetric tensors for the purpose of illustration:

$$
D_{\mu \nu}=\frac{\tilde{u}_{\mu} \tilde{n}_{\nu}-\tilde{n}_{\mu} \tilde{u}_{\nu}}{\sqrt{\tilde{u}^{2} \tilde{n}^{2}}} .
$$

So we will define the self-energy tensor as a sum involving these tensors:

$$
\Pi_{\mu \nu}=a \tilde{\eta}_{\mu \nu}+b L_{\mu \nu}+c n_{\mu \nu}+d S_{\mu \nu}+i d^{\prime} D_{\mu \nu} .
$$

With the velocity 4-vector of the thermal bath defined as in Sec. VA, and the magnetic field in this frame assumed to have the components specified in Sec. VC, we can construct the matrix $\Pi$ easily, using Eq. (83). It will be given by

$$
\boldsymbol{\Pi}=\frac{1}{k^{2}}\left(\begin{array}{cccc}
-\sigma K^{2} & \sigma \omega K & \gamma \frac{K}{\omega} k^{2} & 0 \\
-\sigma \omega K & \sigma \omega^{2} & \gamma k^{2} & 0 \\
-\gamma^{*} \frac{K}{\omega} k^{2} & \gamma^{*} k^{2} & \sigma^{\prime} k^{2} & 0 \\
0 & 0 & 0 & a k^{2}
\end{array}\right) .
$$

where we have used the shorthand

$$
\begin{aligned}
\sigma & =a+b+c \cos ^{2} \alpha+2 d \cos \alpha, \\
\sigma^{\prime} & =a+c \sin ^{2} \alpha, \\
\gamma & =\left[c \cos \alpha+\left(d+i d^{\prime}\right)\right] \tan \beta \cos \alpha .
\end{aligned}
$$

The angle $\alpha$ appearing in these equations is related to the angle $\beta$ that appears in Eq. (72) through the formula

$$
\cos \alpha=\frac{\omega \cos \beta}{\sqrt{\omega^{2} \cos ^{2} \beta+k^{2} \sin ^{2} \beta}} .
$$


One eigenvalue and the corresponding eigenvector is exactly the same as that given in Eq. (75). The other two nonzero eigenvalues satisfy the equation

$$
\Lambda^{2}-\left(\sigma+\sigma^{\prime}\right) \Lambda+\left(\sigma \sigma^{\prime}-|\gamma|^{2} \frac{k^{2}}{\omega^{2}}\right)=0,
$$

so that

$$
\Lambda=\frac{1}{2}\left[\sigma+\sigma^{\prime} \pm \sqrt{\left(\sigma-\sigma^{\prime}\right)^{2}+4\left|\gamma^{2}\right| k^{2} / \omega^{2}}\right] .
$$

The eigenvectors corresponding to these two eigenvalues are of the form

$$
\boldsymbol{\epsilon}_{(a)} \propto\left(\begin{array}{c}
\gamma K \\
\gamma \omega \\
\left(\Lambda_{(a)}-\sigma\right) \omega \\
0
\end{array}\right) \quad \text { for } a=1,2
$$

In fact, the results of both Secs. VA and V C can be seen as special cases of the result given here.

\section{AXISFREE NOTATION}

Throughout Sec. V, we have made specific choices for the directions of the photon momentum and other vectors such as $u^{\mu}$ and $b^{\mu}$. This fact should not be seen as a limitation of the method described. Often, the choices meant no loss of generality. For example, consider the choices made in Sec. V C. We can always choose the axes in such a way that the 3-momentum of the photon is in the $x$-direction, and then define the $x-y$ plane to contain the direction of the magnetic field. There is no loss of generality in assuming the forms given in Eqs. (52) and (72).

If one wants different directions for some reason, one just needs to write the relevant vectors in the appropriate form. For example, if one wants to keep the direction of the photon 3-momentum arbitrary in the coordinate axes, one just needs to redo the exercise by replacing Eq. (52) with a different matrix, whose last three elements should be the Cartesian components of a 3-vector in terms of the magnitude $\kappa$ and the polar and azimuthal angles. It will just make the matrices look a bit more cumbersome, but the task remains the same in principle.

However, it is not difficult to take an alternative route, viz., to write the results in a form that is free from the choices of the axes. All we need to do is to recast the equations in terms of the vectors and tensors that appear in the problem. This was already done for the case of a thermal background, where we saw that the longitudinal polarization vector is just $\tilde{u}^{\mu}$. Here, we outline how such a treatment can be extended to problems with a magnetic field.
The important task is to find a set of four vectors which are linearly independent, and can therefore serve as a basis. One of them is surely $k^{\mu}$, and we define the notation

$$
e_{(0)}^{\mu}=k^{\mu} .
$$

There is no pressing need for normalizing any of the basis vectors, so we will not bother.

\section{A. Background magnetic field}

Here, we need to construct some vectors which are orthogonal to $k^{\mu}$. We can construct a series of vectors, contracting $k^{\mu}$ with different powers of the magnetic field tensor, like

$$
\begin{aligned}
(k \cdot B)^{\mu} & =k_{\lambda} B^{\lambda \mu}, \\
(k \cdot B \cdot B)^{\mu} & =(k \cdot B)_{\lambda} B^{\lambda \mu}=k^{\rho} B_{\rho \lambda} B^{\lambda \mu},
\end{aligned}
$$

and so on. We can use these vectors to form a mutually orthogonal set of vectors. Starting with $k^{\mu}$, we can apply the Gram-Schmidt orthogonalization process to obtain the next two in the set:

$$
\begin{aligned}
& e_{(1)}^{\mu}=k_{\lambda} B^{\lambda \mu} \equiv(k \cdot B)^{\mu}, \\
& e_{(2)}^{\mu}=(k \cdot B \cdot B)^{\mu}+\frac{k^{\mu}}{k^{2}}(k \cdot B)^{2} .
\end{aligned}
$$

We can go on with the next member of the type shown in Eq. (91). But it is much easier to complete the set by introducing the vector

$$
e_{(3)}^{\mu}=\frac{1}{k^{2}} \varepsilon^{\mu \nu \lambda \rho} k_{\nu}(k \cdot B)_{\lambda}(k \cdot B \cdot B)_{\rho},
$$

because for any three 4-vectors $A^{\mu}, B^{\mu}, C^{\mu}$, the object

$$
V^{\mu}=\varepsilon^{\mu \nu \lambda \rho} A_{\nu} B_{\lambda} C_{\rho}
$$

will be orthogonal to all three. The prefactor $1 / k^{2}$ has been put in Eq. (92c) just to ensure that $e_{(3)}^{\mu}$ has the same physical dimension as the other two. It is not really necessary to ensure that, but it is convenient.

We can now see the effect of contracting the self-energy tensor, given in Eq. (71), with these basis vectors. Contraction with $k^{\mu}$ will give zero, of course. For the other ones, we get

$$
\begin{aligned}
& \Pi_{\nu}^{\mu} e_{(1)}^{\nu}=a e_{(1)}^{\mu}, \\
& \Pi_{\nu}^{\mu} e_{(2)}^{\nu}=a e_{(2)}^{\mu}+i b e_{(3)}^{\mu}, \\
& \Pi_{\nu}^{\mu} e_{(3)}^{\nu}=a e_{(3)}^{\mu}+i b \frac{(k \cdot B)^{2}}{k^{2}} e_{(2)}^{\mu} .
\end{aligned}
$$


Equation (94a) shows that $e_{(1)}^{\mu}$ is an eigenvector, with eigenvalue $a$. For the choice of the axes made in Sec. V C, this eigenvector happened to be along the $z$-axis, which is what shows in Eq. (75). The other two eigenvectors are linear combinations of the basis vectors $e_{(2)}^{\mu}$ and $e_{(3)}^{\mu}$. These eigenvectors can easily be found, and the result is

$$
\Lambda_{( \pm)}=a \pm r b, \quad \boldsymbol{\epsilon}_{( \pm)}=\boldsymbol{e}_{(3)} \pm i r \boldsymbol{e}_{(2)}
$$

where

$$
r^{2}=-\frac{(k \cdot B)^{2}}{k^{2}}
$$

Despite the negative sign in this formula, $r$ is a real number, because $k \cdot B$ is a spacelike vector whereas $k$ is timelike. We have not made any effort for writing the eigenvectors in normalized form.

Equations (94a) and (95) give the axisfree definition of the eigenvalues and eigenvectors. With the choices made in Sec. VC, we have $(k \cdot B)^{2}=-K^{2} \sin ^{2} \beta$, and so the eigenvalues shown in Eq. (76) follow. The eigenvectors also reduce to the ones showed there, apart from the overall normalization which has not been adjusted in Eq. (95) anyway.

\section{B. Magnetic field in a thermal medium}

Here also, we can start with the vector $k^{\mu}$. Then, in the list, we can add

$$
e_{(1)}^{\mu}=\tilde{u}^{\mu}
$$

which is orthogonal to $k^{\mu}$. The next one can involve $\tilde{n}^{\nu}$. In order that it is orthogonal to both $e_{(0)}^{\mu}$ and $e_{(1)}^{\mu}$, we choose

$$
e_{(2)}^{\mu}=\tilde{n}^{\mu}-\frac{\tilde{u} \cdot \tilde{n}}{\tilde{u}^{2}} \tilde{u}^{\mu}
$$

And the final one can be chosen as

$$
\epsilon_{(3)}^{\mu}=\varepsilon^{\mu \nu \lambda \rho} k_{\nu} \tilde{u}_{\lambda} \tilde{n}_{\rho}=\varepsilon^{\mu \nu \lambda \rho} k_{\nu} u_{\lambda} n_{\rho}
$$

which is obviously orthogonal to the other ones defined earlier. We do not care about the normalization at this stage, because it is not necessary.

We now contract the self-energy tensor with these basis vectors. It will of course show that $k^{\mu}$ is an eigenvector with zero eigenvalue. On the other three, first of all, we will find one which stands out, viz.,

$$
\Pi^{\mu}{ }_{\nu} e_{(3)}^{\nu}=a e_{(3)}^{\mu} .
$$

It means that $e_{(3)}^{\nu}$ is an eigenvector of the matrix $\Pi^{\mu}{ }_{\nu}$, with eigenvalue $a$. This was the result shown in Eq. (75).

For the other two basis vectors, we get equations of the form

$$
\begin{aligned}
& \Pi^{\mu}{ }_{\nu} e_{(1)}^{\nu}=P_{1} e_{(1)}^{\mu}+Q_{1} e_{(2)}^{\mu}, \\
& \Pi^{\mu}{ }_{\nu} e_{(2)}^{\nu}=P_{2} e_{(1)}^{\mu}+Q_{2} e_{(2)}^{\mu},
\end{aligned}
$$

where

$$
\begin{aligned}
P_{1} & =a+b+c \frac{(\tilde{u} \cdot \tilde{n})^{2}}{\tilde{u}^{2} \tilde{n}^{2}}+2 d \frac{\tilde{u} \cdot \tilde{n}}{\sqrt{\tilde{u}^{2} \tilde{n}^{2}}}, \\
P_{2} & =\frac{\tilde{u}^{2} \tilde{n}^{2}-(\tilde{u} \cdot \tilde{n})^{2}}{\tilde{u}^{4} \tilde{n}^{2}}\left(c \tilde{u} \cdot \tilde{n}+\left(d+i d^{\prime}\right) \sqrt{\tilde{u}^{2} \tilde{n}^{2}}\right), \\
Q_{1} & =\frac{1}{\tilde{n}^{2}}\left(c \tilde{u} \cdot \tilde{n}+\left(d-i d^{\prime}\right) \sqrt{\tilde{u}^{2} \tilde{n}^{2}}\right), \\
Q_{2} & =a+c\left(1-\frac{(\tilde{u} \cdot \tilde{n})^{2}}{\tilde{u}^{2} \tilde{n}^{2}}\right) .
\end{aligned}
$$

This means that the eigenvectors will be linear combinations of $e_{(1)}^{\mu}$ and $e_{(2)}^{\mu}$. If we ignore the task of normalizing the eigenvectors and call an eigenvector $e_{(1)}^{\mu}+r e_{(2)}^{\mu}$, then $r$ will be given by

$$
r=\frac{1}{2 P_{2}}\left(Q_{2}-P_{1} \pm \sqrt{\left(Q_{2}-P_{1}\right)^{2}+4 Q_{1} P_{2}}\right),
$$

and the eigenvalue will be given by

$$
\Lambda=\frac{1}{2}\left[Q_{2}+P_{1} \pm \sqrt{\left(Q_{2}-P_{1}\right)^{2}+4 Q_{1} P_{2}}\right] .
$$

It is easy to see that, with the choice of axes made in Section V, this expression reduces to that in Eq. (88).

\section{COMMENTS}

We started this article by saying that we want to proceed, as much as possible, without committing ourselves to any specific background. We laid down our formalism in Sec. II through Sec. IV, where we showed that the problem can be cast in the form of a problem in matrix algebra. In Sec. V, we illustrated the method with some choices of background and choices of specific axes. In Sec. VI, we went one step further and found the polarization vectors and the dispersion relation in a completely axisfree notation in the cases of a background magnetic field, with or without a background medium.

Of course in the process one has to identify some tensors which are orthogonal to the photon 4-momentum. This part is the same as that in a conventional treatment $[1-4,7]$. The difference in our approach is that, because we identify the polarization vectors as eigenvectors of a matrix, it is easier to find the polarization vectors. Our approach also shows 
that, no matter how many tensors participate in the making of the self-energy, it is guaranteed that one will obtain three polarization vectors, as is expected because the photon is a spin-1 particle.

There is another piece of information that is easily derived from the approach of Sec. VI. This is the question of degeneracy of the dispersion relations. This question is related to the possibility of defining the basis vectors in the matrix formulation. We can always take the photon momentum $k^{\mu}$ as one of the basis vectors. If the background is nontrivial, we will be able to add at least one more vector to this list. That is not enough for lifting degeneracy of the dispersion modes. If we have a third vector, then we can also find a fourth one through the prescription of Eq. (93). But even this is not enough: the four vectors so defined must be linearly independent in order that they can form a basis.

There is an easy check for linear independence. In the demonstrative examples, we always chose the fourth basis vector through Eq. (93). Clearly, this fourth vector cannot be linearly dependent on the other three. We therefore need to check whether the first three basis vectors are linearly independent. If they are dependent, then the vector $V^{\mu}$, defined in Eq. (93), will be the null vector.

Clearly, $e_{(0)}^{\mu}$ cannot be proportional to either $e_{(1)}^{\mu}$ and $e_{(2)}^{\mu}$, because the former is a timelike vector whereas the latter ones are spacelike. Thus, our task reduces to finding whether any combination of $e_{(1)}^{\mu}$ and $e_{(2)}^{\mu}$ can be the null vector. Either any of them will have to be the null vector by itself, or they must be proportional to each other.

For the case dealt with in Sec. VIA, one of these possibilities mean that $k \cdot B$ is itself a null vector. This means that the magnetic field is parallel to the spatial direction of the photon momentum vector. In the notation of Sec. V C, this means that $\beta=0$, and definitely it shows degenerate modes. In the axisfree notation as well, we see that this state of affairs imply $r=0$ in Eq. (96), so that we have degenerate modes. Another alternative, viz. the vanishing of $e_{(2)}^{\mu}$, is impossible because that requires $k \cdot B \cdot B$, a spacelike vector, to be proportional to $k^{\mu}$, a timelike vector.

For the case described in Sec. VI B, the basis vectors are linearly dependent if $\tilde{u}^{\mu}$ and $\tilde{n}^{\mu}$ are proportional. In the notation of Sec. V D, this means that the angle $\beta$ vanishes, and therefore so does $\alpha$ through Eq. (86). Then, from Eq. (85), we find that $\gamma=0$, which means that the eigenvalues obtained from Eq. (88) are $\sigma$ and $\sigma^{\prime}$. But, for $\alpha=0$, we get $\sigma^{\prime}=a$ from Eq. (85), so that this root is degenerate with the root noted down in Eq. (75).

Overall, we find that the matrix formulation of the photon propagation problem clears up many aspects of the polarization vectors and dispersion relations which are otherwise not easy to understand from the usual approach. And, although we used the photon all along to describe the methods, they are applicable to gluons, or any other spin-1 boson for which there is an associated gauge invariance.

\section{ACKNOWLEDGMENTS}

I have had numerous discussions with Munshi Golam Mustafa and Bithika Karmakar during the time they had been working on Ref. [7]. Both of them have also kindly read an earlier version of this article and made helpful comments. At the time of working on the present paper, I have benefitted from discussions with Sumit Das and Ashoke Sen. The work was supported by the SERB research Grant No. EMR/2017/001434 of the Government of India.
[1] M. B. Kislinger and P. D. Morley, Collective phenomena in gauge theories. 1. The plasmon effect for Yang-Mills fields, Phys. Rev. D 13, 2765 (1976).

[2] H. Arthur Weldon, Covariant calculations at finite temperature: The relativistic plasma, Phys. Rev. D 26, 1394 (1982).

[3] J.F. Nieves and P. B. Pal, $P$ and $C P$ odd terms in the photon self-energy within a medium, Phys. Rev. D 39, 652 (1989); Erratum, Phys. Rev. D 40, 2148 (1989).

[4] A. K. Ganguly, S. Konar, and P. B. Pal, Faraday effect: A field theoretical point of view, Phys. Rev. D 60, 105014 (1999).

[5] A. E. Shabad and V. V. Usov, Real and virtual photons in an external constant electromagnetic field of most general form, Phys. Rev. D 81, 125008 (2010).
[6] K. Hattori and K. Itakura, Vacuum birefringence in strong magnetic fields: (I) Photon polarization tensor with all the Landau levels, Ann. Phys. (Amsterdam) 330, 23 (2013).

[7] B. Karmakar, A. Bandyopadhyay, N. Haque, and M. G. Mustafa, General structure of gauge boson propagator and its spectra in a hot magnetized medium, Eur. Phys. J. C 79, 658 (2019).

[8] M. Bordag and V. Skalozub, Polarization tensor of charged gluons in color magnetic background field at finite temperature, Phys. Rev. D 77, 105013 (2008).

[9] K. Hattori and D. Satow, Gluon spectrum in a quark-gluon plasma under strong magnetic fields, Phys. Rev. D 97, 014023 (2018).

[10] M. Nopoush, Y. Guo, and M. Strickland, The static hardloop gluon propagator to all orders in anisotropy, J. High Energy Phys. 09 (2017) 063. 
[11] S. Ghosh, A. Mukherjee, P. Roy, and S. Sarkar, General structure of the neutral $\rho$ meson self-energy and its spectral properties in a hot and dense magnetized medium, Phys. Rev. D 99, 096004 (2019).

[12] For properties of eigensystems of non-normal matrices, see, e.g., P. B. Pal, Algebraic Structures of Physicists
(Cambridge University Press, Cambridge, England, 2019), Sect. 5.10.

[13] J.F. Nieves and P. B. Pal, The third electromagnetic constant of an isotropic medium, Am. J. Phys. 62, 207 (1994). 